\title{
CULTURE OF EMBRYO WITH A SEGMENT OF OVARY IMPROVED GERMINATION AND CROSSABILITY OF DISTANT HYBRIDS IN INDICA RICE
}

\author{
Bindeshwar Prasad Sah*, Raj Kumar Niroula* and Hari Prasad Bimb* \\ *Biotechnology Unit, Nepal Agriculture Research Council, Khumaltar, Lalitpur, Nepal.
}

\begin{abstract}
Distant hybridization in the genus Oryza is realized as an efficient Biotechnological tool for plant breeding work to introgress useful gene/s from diverse array of wild relatives into cultivated rice. This study was carried out to improve the germination frequency of hybrid embryo to enhance the crossability between $O$. sativa sub spp. indica and wild species. Three cultivars of indica rice viz. IR 64, Radha 4 and IR 69618 - CMS A line (cytoplasmic male sterile A line) were pollinated with the pollen of $O$. latifolia, $O$. minuta and $O$. officinalis. Hybrid caryopsis containing embryos were rescued at tenth day of pollination. In vitro germination frequency of rescued embryos were compared by culturing embryo alone and embryo with a bit of ovary during 2005-06 at Biotechnology Unit, Khumaltar, Lalitpur, Nepal. Culture was maintained at $25 \pm 1{ }^{\circ} \mathrm{C}$ under dark until germination and there after continuous light. In majority of the cross combinations, the germination frequencies were found to be higher when embryo excised and cultured with small portion of ovary. This technique yielded up to 100 per cent germination which were later employed to study the crossability between species. Depending upon the cultivars of $O$. sativa, the frequencies of crossability varied from 0.53 to 3.08 per cent with highest for Radha 4/O. minuta. A total of 38 hybrid plants were successfully produced from 88 cultured embryos isolated from 2644 pollinated florets. Inclusion of a bit of ovary along with embryo in in vitro culture was found to be an effective method not only to improve the germination frequency of hybrid embryo, but also to increase the crossability between cultivars of cultivated rice and their distant relatives.
\end{abstract}

Key words: Embryo rescue; Interspecific hybrid; Oryza sativa; Wild species; O. latifolia; O. minuta; O. officinalis.

\section{INTRODUCTION}

Rice is the main source of food for about half of the world's population. It is cultivated in more than 100 countries on every continent except for Antarctica, although 91 percent of the world's rice is grown and consumed in Asia. Globally, rice is the most important crop in terms of its contribution to human diets and value of production. Rice provides 35 to 80 per cent of the calories consumed by 3.3 billion people in Asia, and 8 per cent of food energy for 1 billion people in sub-Saharan Africa and Latin America and the Caribbean. In 2005, the global area harvested to rice was approximately 154 million hectares with almost 621 million tones of production. The area harvested in Asia was 137 million hectares producing 562 million tones where as almost 9 million hectares with production of 19 million tones in Africa. A total of 4.2 million tones of rice were produced in the same year from 1.55 million hectares with $2717 \mathrm{~kg} / \mathrm{ha}$ of yield in Nepal.

Cultivated rice belongs to two species, Oryza sativa (which is more widely used) and Oryza glaberrima- an African rice. The two main strains of $O$. sativa are japonica and indica. The differences between these two evolved both geographically and culturally over thousands of years as farming groups relocated to different ecosystems. Some groups moved to new land at higher elevations, while others established lowland rice fields in dry areas, seasonally flooded ground, or tidal swamp areas. Over the millennia, different types of rices evolved that were cultivated under different ecological conditions. Today, there are thousands of varieties of japonica and indica rice grown in more than 100 countries.

There was a notable jump in the yield of rice during the beginning of 1960's. International Rice Research Institute, Manila, Philippines has made a major contribution in the manipulation of genetic background of cultivated rice. Recently, the cultivated rice has shown the plateauing of yield in rice growing areas of the world. The yield level of rice in the rice growing countries of the world including Nepal is comparatively very low. The major causes of lower yield of cultivated rice are susceptible to major biotic and abiotic stresses. Several wild relatives of rice have been studied in the past and found to possess valuable genes of resistance for above stresses (Brar, 2005).

Distant hybridization in cereal is a significant Biotechnological tool for plant breeding work for the incorporation of desirable genes from wild species into cultivated ones. Several pre and

Author for Correspondence: Bindeshwar Prasad Sah, Biotechnology Unit, Nepal Agriculture Research Council, Khumaltar, Lalitpur, Nepal.Email: bprasadsah@rediffmail.com. 
post fertilization barriers have been reported to restrict the production of wide hybrids, and subsequent gene introgression work (Williams et al., 1987). The genus Oryza has more than 22 wild species. Based on the restriction and ease of gene transfer, the wild gene pools have been grouped into three complexes namely, primary (sativa complex), secondary (officinalis complex) and tertiary (other complex) (Khush, 2000). The production of distant hybrids from crosses between cultivated and wild species of rice belong to secondary and tertiary pools and are extremely difficult because of the premature death of embryo. The earlier abortion of hybrid embryo at different developmental stage is the characteristic feature in rice (Brar and Khush, 1997). Embryo rescue technique is developed to facilitate the successful distant hybridization. Hence, the transfer of genes from wild to cultivated rice can be achieved following the sequential steps viz. manual hybridization, embryo rescue, backcrossing, and selection processes (Jena and Khush, 1986).

Embryo rescue is a handy tool in which inherently weak, immature or hybrid embryos prior to degeneration are aseptically excised and cultured in vitro. Niles (1951) was the first who initiated in vitro germination in rice. Since then several refinements have been made in this technique and has become a popular approach for raising the distant rice hybrids from a number of incompatible crosses (Iyer and Govila, 1964; Ko et al., 1983; Jena and Khush, 1984; Bouharmont, 1991; Brar et al., 1991). Jena and Khush (1984) have demonstrated the potential role of embryo rescue for the production of distant hybrids in the genus Oryza. Following this technique, several resistant genes such as grassy stunt, bacterial blight, blast, brown plant hopper, whitebacked planthopper, and root-knot nematode, tolerance to tungro, CMS (cytoplasmic male sterility) and acid sulfate soil have been transferred to cultivated rice. Further, several other traits like yield, earliness etc. have been improved by introducing genes from wild rice species (Brar, 2005). In most of these works, the germination frequencies of hybrid embryos were very low. Therefore, this study was carried out with the following objectives: 1) to study the germination frequency of isolated embryos, and 2) to produce the intergenomic rice hybrids.

\section{MATERIALS AND METHODS}

The study was carried out with three wild species viz. $O$. latifolia (2n = 48, CCDD, IRGC Acc. 100914), O. minuta (2n = 48, BBCC, IRGC Acc. 10114), and O. officinalis ( $2 n=24$, CC, NPGR Acc. 10602), and three cultivated rice viz. IR 64, Radha 4 and CMS IR 69618-A. The wild species were used as male parents while cultivated as female. Seeds of $O$. latifolia, $O$. minuta, and CMS IR 69618-A were received from International Rice Research Institute, Los Banos, Laguna, Philippines. Seeds of $O$. officinalis were collected from Agriculture Botany Division, NARC, Khumaltar, Lalitpur, Nepal. Due to the poor germination and strong dormancy, collected seeds of respective species were germinated in vitro by culturing dehulled sterilized seeds in $1 / 4^{\text {th }}$ MS basal medium and incubated in dark at $25 \pm 1^{\circ} \mathrm{C}$ until germination. Once the seeds were germinated, seedlings were kept under continuous light illuminated with fluorescent light in 16/8 h day-night regimes for 2-3 leaf stage. Fully grown seedlings were hardened and planted into three plastic buckets filled with well fertilized sterilized soils. Staggered plantings of female parents were made continuously at four days of intervals to synchronize the flowering time. Each female seed was planted in two replications; two buckets/replication, and two seeds/bucket at a time. All agronomic practices were practiced to raise the healthy parents. Each female parent was crossed with each of wild species. Florets of female parents except CMS line were hand emasculated at one to two days prior to anthesis. During anthesis, pollen of each wild species were collected separately in petridish and pollinated immediately with the help of fine brush. Each floret was pollinated repeatedly for 2-3 days.

After nine days of pollination, seed settings were recorded by counting spikelets with and without developed caryopsis. Before excision, the spikelets without seed set were removed from the panicle and remaining whole panicles were immersed in $70 \%$ ethanol for one minute and finally sterilized in $2 \%$ sodium hypochlorite solution for 20 minutes. The spikelets were detached from sterilized panicles and washed thrice thoroughly in sterile distilled water. Embryos were isolated in two different ways to compare the germination frequency as: i) only naked embryos, and ii) a segment of ovary containing embryo. Naked embryos were isolated by clipping spikelets at 2-3 $\mathrm{mm}$ above the pedicel end and squeezing the embryo out of the floret using a pair of sterile forceps. A part of ovary containing embryo and/or endosperm was also prepared by using same procedure but the embryo was not removed completely from the ovary. The extra upper part of the caryopsis was then trimmed by leaving $2 \mathrm{~mm}$ segment using sterile scissors and forceps. In both methods, 10 days old embryos prior to abort were rescued and cultured in vitro. Observations on frequency (\%) of and incubation period (day) required for germination in both methods were recorded separately by rescuing 45 embryos per treatment in each of five crosses. Such embryos were inoculated on $1 / 4^{\text {th }} \mathrm{MS}$ basal medium (Murashige and Skoog, 1962). The medium was gelled by Agar $(0.7 \%)$ after adjusting $\mathrm{pH}$ at 5.8. Cultures were maintained in a temperature-controlled chamber at $25 \pm 1^{\circ} \mathrm{C}$ under dark until germination and then continuous fluorescent light. After three weeks of culture, the frequency of germination was recorded at three days interval up to $15^{\text {th }}$ day. The germinated embryos from both methods were counted and frequency was determined in percentage.

Based on the germination frequency, crossability study was carried out using the product of second method. After five weeks of incubation, regenerated seedlings at three-leaf stage were removed from the culture tubes. Roots of the seedlings were thoroughly washed in tap water to remove media and finally washed with sterilized distilled water. The hardened seedlings were planted in plastic buckets filled with sterilized soil and grown in the glasshouse during normal and winter seasons of 2005-2006. Crossability of each hybrid was recorded and frequency was determined as (number of $\mathrm{F}_{1}$ 
plants obtained/total florets pollinated) x 100 after confirming the hybrid plants during 2006 (Wuu et al., 1963). Morphological characters of regenerated hybrids and their respective parents were recorded for their comparison.

\section{RESULTS AND DISCUSSION}

All the resultant hybrid seeds were lack of endosperm and only filled with the watery fluid. Embryo degeneration phenomenon was observed across the combinations, and that was successfully overcomed by applying the embryo rescue technique (Fig. 3, 4 \& 5). Between two methods of embryo culture, a segment of ovary containing embryo showed higher germination frequency with better root and shoot growth than the culture of naked embryo alone (Fig. 1, 3, 4 \& 5). The germination frequency varied in both treatments depending upon the cross combinations. The germination frequency in naked embryo culture ranged from 20 to 60 per cent where as it was 40 to 100 per cent in culture of embryo with a part of ovary. The effect of segment of ovary on germination was observed only during initial days of incubation (Fig. 2). After nine days of incubation, the second method showed higher response and the frequency of germination was recorded as doubled at $15^{\text {th }}$ day of incubation. The growth of seedlings from the second method was also better. Following this second technique, 38 hybrid plants were successfully produced from 88 cultured embryos isolated from 2644 pollinated florets. In this experiment, the frequency of germination ranged from 43.75 to 83.33 per cent and crossability from 0.53 to 3.08 per cent (Table 1 ). The highest (3.08\%) and lowest $(0.53 \%)$ crossability was recorded for Radha 4/O. minuta and IR 64/O.officinalis, respectively. Majority of the regenerated $\mathrm{F}_{1}$ hybrids were found to be male sterile. In general, the morphology of hybrids was vigorous and intermediate to both parents (Fig. 6).

The successful production of hybrid plants from the cultured embryos were observed to be largely dependent upon the maturation stage, the technique of embryo excision, composition of the culture medium, and several other factors associated with culture conditions (Iyer and Govila, 1964; Sharma et al., 1995). Excision of uninjured embryo is necessary for higher frequency of germination. The higher frequency of germination obtained in the second method might be due to the culture of uninjured embryo rather than other factors. In the second method, complete dissection of ovary was not

Table 1: Response of culture of embryo with a segment of ovary to germination and crossability.

\begin{tabular}{|c|c|c|c|c|c|c|c|c|}
\hline \multirow[t]{2}{*}{ Cross combination } & \multirow[t]{2}{*}{$\begin{array}{c}\text { Florets } \\
\text { pollinated }\end{array}$} & \multirow[t]{2}{*}{$\begin{array}{c}\text { Seed } \\
\text { set }\end{array}$} & \multicolumn{2}{|c|}{$\begin{array}{c}\text { Seed without } \\
\text { embryo }\end{array}$} & \multirow[t]{2}{*}{$\begin{array}{c}\text { Embryos } \\
\text { cultured }\end{array}$} & \multirow{2}{*}{\begin{tabular}{|c|} 
Germination \\
$(\%)$ \\
\end{tabular}} & \multirow[t]{2}{*}{$\begin{array}{c}F_{1} \text { hybrid } \\
\text { plants }\end{array}$} & \multirow{2}{*}{$\begin{array}{c}\text { Crossability } \\
(\%)\end{array}$} \\
\hline & & & & $(\%)$ & & & & \\
\hline IR 64/O. latifolia & 248 & 19 & 10 & 52.63 & 9 & 66.66 & 4 & 1.61 \\
\hline Radha 4/O. latifolia & 205 & 22 & 11 & 50.00 & 11 & 54.55 & 5 & 2.44 \\
\hline CMS/O. latifolia & 226 & 15 & 2 & 13.33 & 10 & 60.00 & 2 & 0.88 \\
\hline IR 64/O. minuta & 275 & 9 & 4 & 44.44 & 5 & 80.00 & 4 & 1.45 \\
\hline Radha 4/O. minuta & 260 & 29 & 13 & 44.83 & 16 & 62.50 & 8 & 3.08 \\
\hline CMS/O. minuta & 525 & 20 & 9 & 45.00 & 11 & 72.73 & 6 & 1.14 \\
\hline IR 64/O. officinalis & 190 & 5 & 1 & 20.00 & 4 & 50.00 & 1 & 0.53 \\
\hline Radha 4/O. officinalis & 218 & 6 & 0 & 0.00 & 6 & 83.33 & 3 & 1.38 \\
\hline CMS/O. officinalis & 497 & 19 & 3 & 15.79 & 16 & 43.75 & 5 & 1.01 \\
\hline Total & 2644 & 144 & 53 & - & 88 & - & 38 & - \\
\hline
\end{tabular}

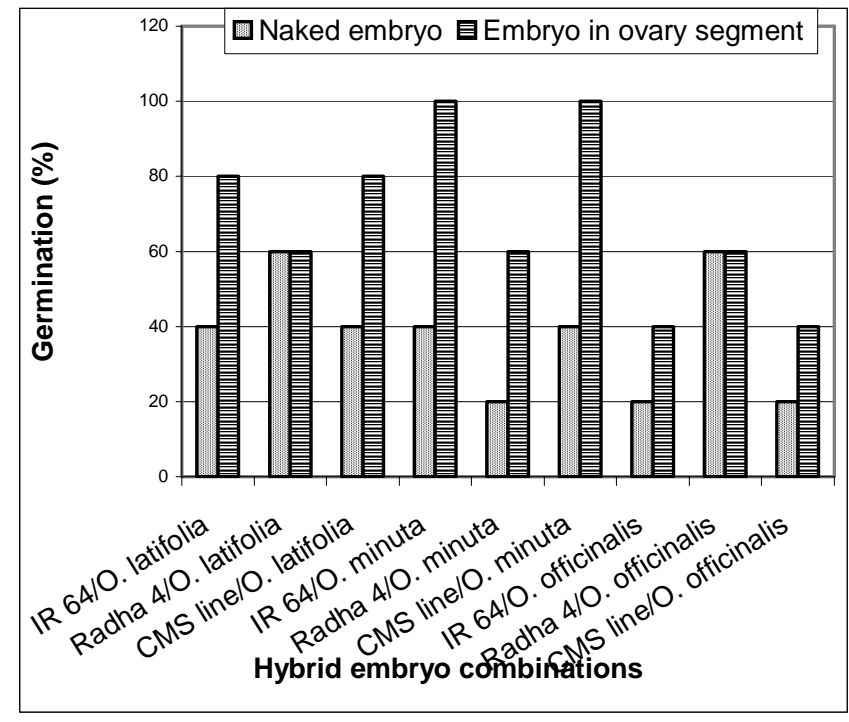

Figure 1: Germination of the interspecific hybrids from naked embryo and a bit of ovary containing embryo.

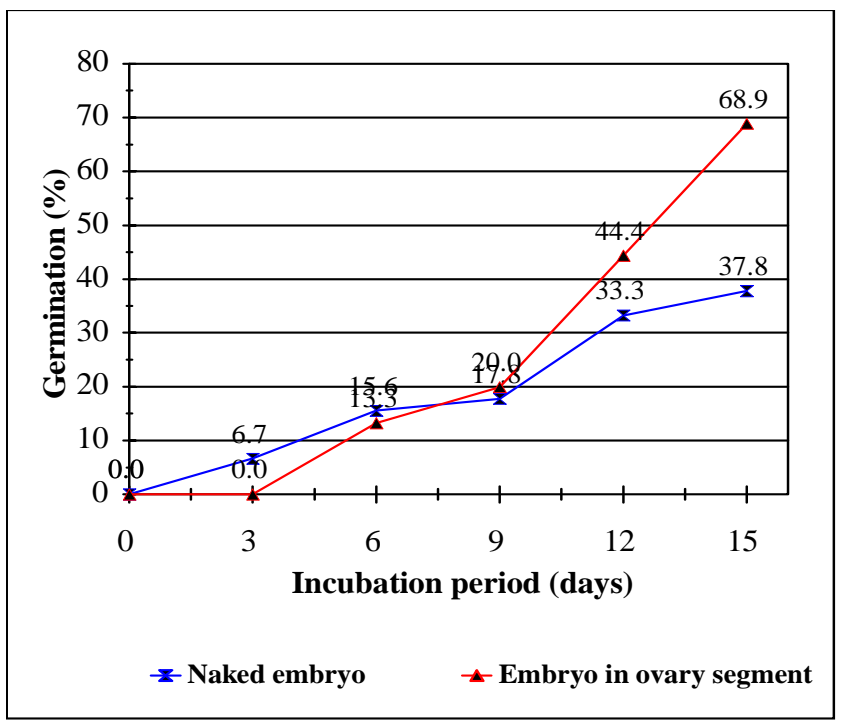

Figure 2: Comparison of methods of culture for germination frequency over various incubation periods. 
made. Therefore, it saved from the accidental injury of embryo during isolation. The isolation of naked embryo requires frequent dissecting of ovary, which can cause accidental injury. Further, forceps sterilized by flaming may be sometimes not properly cooled, and hence, can burn the outer structural integrity of embryo. Such injured embryos could not get germinated in in vitro culture even if other factors are conducive. The second technique also facilitated the rapid isolation of uninjured embryo since it does not require any dissecting work under microscope. Similar results were reported by Iyer and Govila (1964). They stated that embryo with a bit of endosperm facilitates safe excision of embryo even though it sometimes delayed the germination. We have also observed that a segment of ovary containing embryo germinate slowly. The delay in germination through the second method might be due to lack of direct contact of embryo with nutrient medium. A segment of ovary needs few days to absorb the required nutrients from the media. In wide hybridization programme, higher frequency of regenerated hybrids is required irrespective of duration for incubation of embryo for germination in in vitro. The similar germination frequency obtained from the cross combinations between Radha 4 with $O$. latifolia and $O$. officinalis might be due to already presence of some wild genes of $O$. nivara in Radha 4. However, this can be reconfirmed by increasing the sample size of embryos in in vitro culture. In the present study, the frequency of germination was higher as compared to the results of others (Jena and Khush, 1984). In the past, Sitch et al. (1989) observed 0 to 80 per cent of germination depending upon the varieties of $O$. sativa and wild speices.

The observed frequency of crossability in this study was also comparable to others (Jena and Khush, 1986; Brar et al., 1991). However, Sitch et al. (1989) obtained 4.1 and 0.53 per cent of crossability, respectively for IR 64/O. latifolia and IR 31917/O. minuta in contrast to the present study (Table 1). The slight discrepancy observed in the present study and the report of others for seed set to plant establishment indicated that the degree of incompatibility is varying with genotypes of $O$. sativa and ecotype of wild species used. There is no evidence to such slight discrepancy obtained in this study and that results found by earlier investigators, except differences in strains used in crossing programme. The crossability between species can be enhanced through the manipulation of various factors from initial hybridization to different aspects of embryo culture.

\section{SUMMARY}

Culture of embryo with a segment of ovary is found to be an efficient technique for producing distant hybrids in rice and can be successfully used even in a simple laboratory conditions. (Figure 3-5 and 6)

\section{ACKNOWLEDGEMENTS}

Dr. D.S. Brar, International Rice Research Institute, LosBanos, Laguna, Philippines is highly appreciated for providing seeds of wild rice.

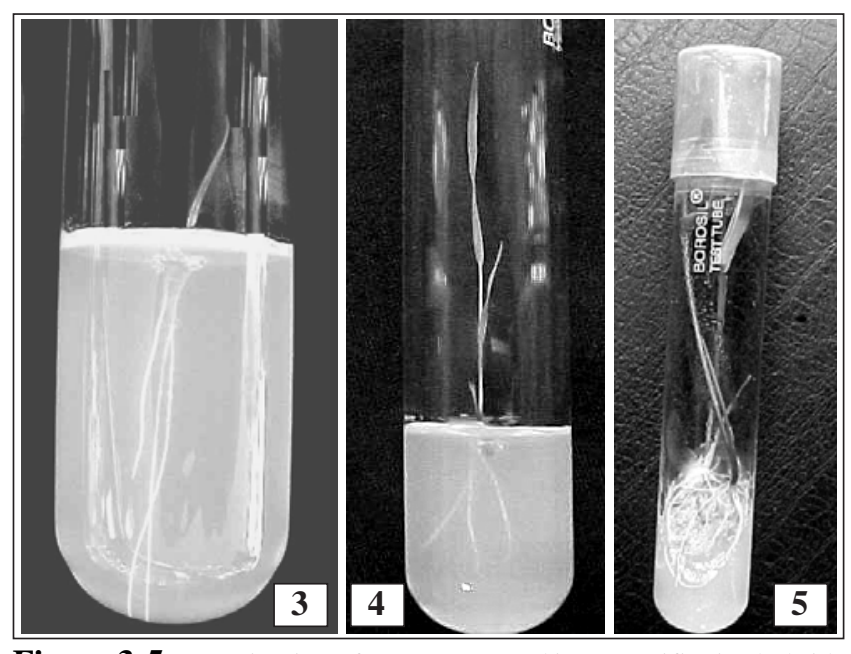

Figure 3-5: Germination of In vitro rescued interspecific rice hybrids after 10 days of incubation: 3. O. sativa cv. Radha 4/O. minuta via. naked embryo culture; 4 . O. sativa cv. Radha 4/O. minuta via a bit of ovary containing embryo; 5 . O. sativa cv. IR 64/O. latifolia via a bit of ovary containing embryo.

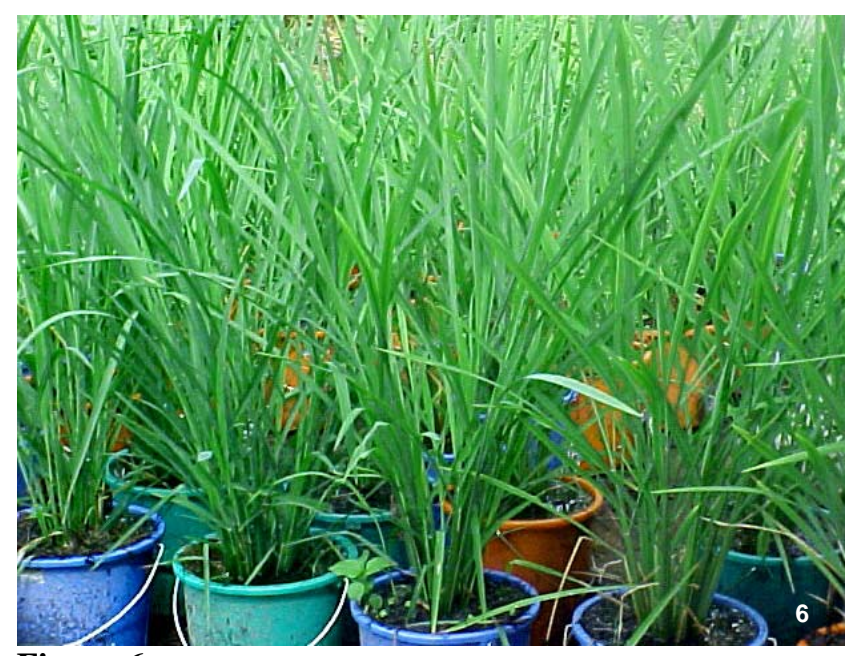

Figure 6: Morphology of well growing interspecific hybrid plants derived from $O$. sativa cv. Radha-4/O. officinalis.

\section{REFERENCE}

Bouharmont, J. 1991. Embryo culture for wide hybridization in rice. In Y. P. S. Bajaj (eds.): Biotechnology in Agriculture and Forestry 14, Rice. Springer-verlag. 95-104.

Brar, D.S. 2005. Broadening the gene pool of rice through introgression from wild species. In K. Toriyama, K.L. Heong and B. Hardy (eds.) : Rice is life: scientific perspectives for the 21st century. 157-160.

Brar, D.S. and Khush, G.S. 1997. Alien introgression in rice. Plant Mol. Biol. 35: 35-47.

Brar, D.S., Elloran, R. and Khush, G.S. 1991. Interspecific hybrids produced through embryo rescue between cultivated and eight wild species of rice. Rice Genet. Newsl. 8: 91-92.

Iyer, R.D. and Govila, O.P. 1964. Embryo culture of interspecific hybrids in the genus Oryza. Indian J. Genet. Plant Breed. 24:116-121.

Jena, K.K. and Khush, G.S. 1984. Embryo resuce of interspecific hybrids and its scope in rice improvement. Rice Genet. Newsl. 1:133-134. 
Jena, K.K. and Khush, G.S. 1986. Production of monosomic alien addition lines of $O$. sativa having single chromosome of $O$. officinalis. In Proceedings of the International Rice Genetics Symposium 27-31, May 1985. Rice Genetics. IRRI, Manila, Philippines. 199-208.

Khush, G.S. 2000. Taxonomy and origin of rice. In R.K Singh, U.S. Singh and G.S. Khush (eds.). Aromatic Rices. 5-13.

Ko, S.W., Wong, C.K. and Woo, S.C. 1983. A simplified method of embryo culture in rice of Oryza sativa L. Bot. Bull. Acad. Sin. 24: 97-101.

Murashige, T. and Skoog, F. 1962. A revised medium for rapid growth and bioassays with tobacco tissue cultures. Plant Physiol. 39: 375-383.

Niles, J. J. 1951. Hybridization methods with paddy. Trop. Agriculturist. 107: 25-29.
Sharma, D.R., Kaur, R. and Kumar, K. 1995. Embryo resuce in plantsa review. Euphytica. 89: 325-337.

Sitch, L.A., Dalmacio, R.D. and Romero, G.O. 1989. Crossability of wild Oryza species and their potential use for improvement of cultivated rice. Rice Genet. Newsl. 6: 58-60.

Williams, E.G., Maheswaran, G. and Hutchinson, J.E. 1987. Embryo culture and ovule culture in crop improvement. Plant Breed. Rev. 5: 181-235.

Wuu, K.D., Jui, Y., Lu, KC.L., Chou, C. and Li, H.W. 1963. Cytogenetical studies of Oryza sativa L. and its related species. 3. Two intersectional hybrids, O. sativa x O. brachyantha A. Chev. et Rohr and O. minuta x O. Brachyantha A. Chev. et Rohr. Bot. Bull. Acad. Sin. 4: 51-59. 